\title{
Provably Secure Tripartite Password Protected Key Exchange Protocol Based on Elliptic Curves ${ }^{\star}$
}

\author{
Sanggon Lee ${ }^{1}$, Yvonne Hitchcock ${ }^{2, \star \star}$, Youngho Park ${ }^{3}$, and Sangjae Moon ${ }^{4}$ \\ 1 Division of Internet Engineering, Dongseo University, \\ Busan 617-716, Korea \\ nok60@dongseo.ac.kr \\ 2 Information Security Institute, Queensland University of Technology, \\ GPO Box 2434 BRISBANE 4001 Australia \\ hitchcock@isrc.qut.edu.au \\ 3 School of Electronics and Electrical Engineering, \\ Sangju National University, Sangju-si, Gyeongsangbuk-do 742-771, Korea \\ yhpark@sangju.ac.kr \\ 4 School of Electrical Engineering and Computer Science, \\ Kyungpook National University, Daegu 702-701, Korea \\ sjmoon@ee.knu.ac.kr
}

\begin{abstract}
Joux's tripartite key agreement protocol is one of the most prominent developments in the area of key agreement. Although certificate-based and ID-based authentication schemes have been proposed to provide authentication for Joux's protocol, no provably secure password-based one round tripartite key agreement protocol has been proposed yet. We propose a secure one round password-based tripartite key agreement protocol that builds on Joux's protocol and adapts PAKEC scheme for password-based authentication, and present a proof of its security.
\end{abstract}

Keywords: Tripartite key agreement; password-based authentication; provable security; bilinear Diffie-Hellman problem; Joux's protocol.

\section{Introduction}

A Key agreement protocol is the mechanism by which two or more parties can establish a common secret key over a network controlled by an adversary. This secret key is commonly called a session key and can then be used to create a secure communications channel among the parties.

The situation where three or more parties share a key is often called conference keying. The three-party (or tripartite) case is of the most practical importance, not only because it is the most common size for electronic conferences, but also

* This work was supported by University IT Research Center Project of MIC, Korea.

** Research funded by Australian Research Council through Discovery Project DP0345775. 
because it can be used to provide a range of services for two communicating parties. For example, a third party can be added to chair, or referee a conversation for ad hoc auditing. Also, a three-party key agreement protocol can be used for tree based group key agreement protocols [12].

Joux's tripartite key agreement protocol [10] is one of the most prominent developments in the area of key agreement. This protocol makes use of pairings on elliptic curves and requires each entity to transmit only a single broadcast messages. This should be contrasted with the obvious extension of the DiffieHellman protocol to three parties, which requires two broadcasts per entity. However, like the basic Diffie-Hellman protocol, Joux's protocol also suffers from man-in-the-middle attacks because it does not provide key authentication.

To transform Joux's protocol into a secure tripartite protocol that only requires one round, many protocols have been proposed, both certificate-based [1,17] and ID-based [18. Another method of authentication is to make use of a password [4, 15, 16]. Certificate-based authentication requires a certificate authority and ID-based authentication requires a trusted dealer with a universal secret key. However, password based authentication does not require any trusted third party. No provably secure password-based one round tripartite key agreement protocol has been proposed so far in the literature.

PAK-EC [15] is a two party password authenticated key agreement protocol built on elliptic curves. Our contribution is to present a provably secure one round password-based tripartite key agreement protocol that builds on Joux's protocol and adapts the PAK-EC scheme for password-based authentication.

In Sect. 2, we describe the model used in the security proof, and in Sect. 3 , the proposed protocol is described. In Sect. 4, we prove the protocol secure, and in Sect. 5] we compare the efficiency of our protocol with another one.

\section{Security Model}

For our proof of security we use the model of Bellare, Pointcheval and Rogaway [3] (which is used by Kate et al. [1] and MacKenzie [16]), and adopt MacKenzie's approach [16]. Our model is for implicitly authenticated key exchange between parties $A, B$ and $C$ who share a secret. The goal is for them to engage in a protocol such that after the protocol is completed, they each hold a session key that is known to nobody but the three of them. In the following, we will describe our model.

Let $I$ be a nonempty set of participants. We assume each participant $U \in I$ is labeled by a string, and we simply use $U$ to denote this string. We will also use $A, B, C, \ldots$ to refer to protocol participants. Each group of three participants, $A, B, C \in I$, who will set up a secret key shared amongst themselves are assumed to share a secret password with each other, $\pi_{A B C}$, before the protocol begins.

For a protocol $P$, each participant is able to execute $P$ multiple times with different partners, and we model this by allowing unlimited number of instances of each participant. Instance $i$ (or session number $i$ ) of participant $U \in I$ is denoted $\prod_{i}^{U}$. To describe the security of the protocol, we assume there is an adversary 
$\mathcal{A}$ that has complete control over the environment (mainly the network), and thus provides the input to instances of participants. Formally, the adversary is a probabilistic algorithm with a distinguished query tape. Participants respond to queries written to this tape according to $P$; the allowed queries are based on and extend the model of Bellare et al. 3. Oracles exist in one of several possible states: Accept, Reject, or * . The state* means no decision has yet been reached. In our protocol, an oracle accepts only after receipt of two correctly formatted messages from the two other participants with whom the oracle wishes to establish a shared key, and the transmission of one message. When an oracle accepts, we assume it accepts holding key $K$ that is $\kappa$ bits in length.

Send $(U, i, M)$ : Causes message $M$ to be sent to instance $\prod_{i}^{U}$. The instance computes what the protocol says to, the oracle's state is updated, and any outgoing messages are given to $\mathcal{A}$. If this query causes $\prod_{i}^{U}$ to accept or terminate, this will also be shown to $\mathcal{A}$. To initiate a session between three participants, the adversary should send a message containing the names of two participants to an unused instance of the other participant.

$\operatorname{Execute}(A, i, B, j, C, l)$ : Causes $P$ to be executed to completion between $\prod_{i}^{A}, \prod_{j}^{B}$ and $\prod_{l}^{C}$ (where $A, B, C \in I$ ), and outputs the transcript of the execution. This query captures the intuition of a passive adversary who simply eavesdrops on the execution of $P$.

$\operatorname{Reveal}(U, i)$ : Causes the output of the session key held by $\prod_{i}^{U}$.

$\operatorname{Test}(U, i)$ : Causes $\prod_{i}^{U}$ to flip a bit $b$. if $b=1$ the session key $s k_{U}^{i}$ is output; otherwise, a string is drawn uniformly from the space of session keys and output. A Test query may be asked at any time during the execution of $P$, but may only be asked once.

$\operatorname{Corrupt}(U)$ : This query returns any passwords that $U$ holds.

Partnering: A participant instance that accepts holds a partner-id pid, sessionid $s i d$, and a session key $s k$. Then instances $\prod_{i}^{A}, \prod_{j}^{B}$, and $\prod_{l}^{C}$ (where $A, B, C$ $\in I)$ are said to be partnered if all of them accept, they hold $\left(\right.$ pid $\left._{A}, s i d_{A}, s k_{A}\right)$, $\left(p_{i d_{B}}, s i d_{B}, s k_{B}\right)$ and $\left(p^{2} d_{C}, s i d_{C}, s k_{C}\right)$, respectively, with $p^{2} d_{A}=\langle B, C\rangle$, $\operatorname{pid}_{B}=\langle A, C\rangle, \operatorname{pid}_{C}=\langle A, B\rangle, \operatorname{sid}_{A}=s i d_{B}=s i d_{C}$, and $s k_{A}=s k_{B}=s k_{C}$, and no other instance accepts with session-id equal to $\operatorname{sid}_{A}, \operatorname{sid}_{B}$ or $\operatorname{sid}_{C}$.

Freshness: We define two notions of freshness, as in [3. Specifically, an instance $\prod_{i}^{U}$ is nfs-fresh (fresh with no requirement for forward secrecy) unless either (1) a $\operatorname{Reveal}(U, i)$ query occurs, $(2)$ a $\operatorname{Reveal}\left(U^{\prime}, j\right)$ query occurs where $\prod_{j}^{U^{\prime}}$ is a partner of $\prod_{i}^{U}$, or (3) a $\operatorname{Corrupt}\left(U^{\prime}\right)$ query occurs for any party $U^{\prime}$ (for convenience, when we do not make a requirement for forward secrecy, we simply disallow Corrupt queries). An instance $\prod_{i}^{U}$ is fs-fresh (fresh with forward secrecy) unless either (1) a $\operatorname{Reveal}(U, i)$ query occurs, (2) a $\operatorname{Reveal}\left(U^{\prime}, j\right)$ query occurs where $\prod_{j}^{U^{\prime}}$ is the partner of $\prod_{i}^{U}$, or (3) a $\operatorname{Corrupt}\left(U^{\prime}\right)$ query occurs for any party $U^{\prime}$ before the Test query and a $\operatorname{Send}(U, i, M)$ query occurs for some string $M$.

We now formally define the authenticated key exchange (ake) advantage of the adversary against protocol $P$. Let $\operatorname{Succ}_{P}^{a k e}(\mathcal{A})$ be the event that $\mathcal{A}$ makes a 
single Test query directed to some fresh instance $\prod_{i}^{U}$ that has terminated, and eventually outputs a bit $b^{\prime}$, where $b^{\prime}=b$ for the bit $b$ that was selected in the Test query. The ake advantage of $\mathcal{A}$ attacking $P$ is defined to be

$$
\operatorname{Adv} v_{P}^{a k e}(\mathcal{A}) \stackrel{\text { def }}{=} 2 \operatorname{Pr}\left[\operatorname{Succ}_{P}^{a k e}(\mathcal{A})\right]-1 .
$$

The following fact is easily verified.

Fact 1. $\operatorname{Pr}\left(\operatorname{Succ}_{P}^{a k e}(\mathcal{A})\right)=\operatorname{Pr}\left(\operatorname{Succ}_{P^{\prime}}^{a k e}(\mathcal{A})\right)+\varepsilon \Leftrightarrow A d v_{P}^{a k e}(\mathcal{A})=A d v_{P^{\prime}}^{a k e}(\mathcal{A})+2 \varepsilon$.

\section{Password and Pairings-Based Tripartite Key Exchange}

We briefly describe some background on pairings on elliptic curves and the BDH assumption, and then present our new tripartite PPK (password protected key exchange) protocol based on Joux's protocol [10].

\subsection{Bilinear Pairings and the BDH Assumption}

We use the same notation as in [5]. Let $G_{1}$ be a cyclic additive group generated by $Q$, whose order is a prime $q$, and $G_{2}$ be a cyclic multiplicative group of the same order $q$. We assume that the discrete logarithm problem (DLP) in both $G_{1}$ and $G_{2}$ is hard. Let $e: G_{1} \times G_{1} \rightarrow G_{2}$ be a pairing which satisfies the following conditions (where $W, X, Z \in G_{1}$ ):

1. Bilinear: $e(W, X+Z)=e(W, X) \cdot(W, Z)$ and $e(W+X, Z)=e(W, Z) \cdot(X, Z)$;

2. Non-degenerate: $e(Q, Q)$ is a generator of $G_{2}$;

3. Computability: $e(P, Q)$ can be efficiently computed for all $P, Q \in G_{1}$.

The Weil or Tate pairing on an elliptic curve can be used to derive $e$ [8, 14.

Definition 1 (Bilinear Diffie-Hellman (BDH) Assumption). Let $G_{1}$ and $G_{2}$ be as defined above with generators $Q$ and $e(Q, Q)$ respectively. Let $\operatorname{ACCEPTABLE}(v)$ be a function that returns true if and only if $v \in G_{1}$. For three values $X, Y$, and $Z$, if $\operatorname{ACCeptable}(Y)$, and $\operatorname{ACCePtable}(Z)$, and $X=a Q$, let $\mathbf{B D H}(X, Y, Z)=\mathbf{B D H}(X, Z, Y)=\mathbf{B D H}(Y, X, Z)=\mathbf{B D H}(Y, Z, X)=$ $\mathbf{B D H}(Z, X, Y)=\mathbf{B D H}(Z, Y, X)=e(Y, Z)^{a}$. (If $X=a Q, Y=b Q$, and $Z=c Q$, then by the definition $\mathbf{B D H}(X, Y, Z)=e(Q, Q)^{a b c}$.) Let $\mathcal{D}$ be an algorithm with input $(X, Y, Z)$. Let

$$
\begin{aligned}
A d v_{G_{1} G_{2}}^{B D H}(\mathcal{D}) \stackrel{\text { def }}{=} \operatorname{Pr}\left[(a, b, c) \stackrel{R}{\leftarrow} Z_{q}^{*} ; X \leftarrow a Q ; Y \leftarrow b Q ; Z \leftarrow c Q\right. \\
: \operatorname{BDH}(X, Y, Z) \in \mathcal{D}(X, Y, Z)]
\end{aligned}
$$

Let $A d v_{G_{1} G_{2}}^{B D H}(t, n)=\max _{\mathcal{D}}\left\{A d v_{G_{1} G_{2}}^{B D H}(\mathcal{D})\right\}$, where the maximum is taken over all adversaries of time complexity at most that output a list containing at most $n$ elements of $G_{2}$. The BDH assumption states that for $t$ and $n$ polynomial in the security parameter $\kappa, A d v_{G_{1} G_{2}}^{B D H}(t, n)$ is negligible. 


\subsection{One Round Tripartite PPK Based on Joux's Protocol}

Figure 1presents a new tripartite PPK protocol based on Joux's protocol. In the protocol, $f_{i}(A, B, C, \pi)$ is defined to be a function generating a random point on elliptic curve $E$ from $A, B, C$, and $\pi$, as specified by MacKenzie [15, who adapted it from IEEE Standard 1363 [9, Appendix A.11.1].

The protocol can be converted to one round by having $A, B$ and $C$ compute and broadcast $m, \mu$ and $v$ respectively to begin the protocol. Each party then waits for the other two parties' messages and carries out its remaining steps (i.e. $B$ and $C$ now carry out their protocol steps in the same order as is currently specified for $A$ ). Such a reordering does not affect the security proof.

We use the terminology "in a Participant $U$ ACtion $i$ query to $\prod_{j}^{U}$ " to mean "in a Send query to $\prod_{j}^{U}$ that results in the Participant $U$ Action $i$ procedure being executed." The possible actions with their associated inputs and outputs are shown in Table 1, where $A$ is the initiator, $B$ is the second participant and $C$ is the third participant.

Precomputation by each party:

$$
\lambda_{A}=r \cdot f_{1}(A, B, C, \pi) ; \lambda_{B}=r \cdot f_{2}(A, B, C, \pi) ; \lambda_{C}=r \cdot f_{3}(A, B, C, \pi) .
$$

Abbreviations: $\mathrm{ACC}=$ ACCEPTABLE; $\quad$ pid $=$ partner ID.

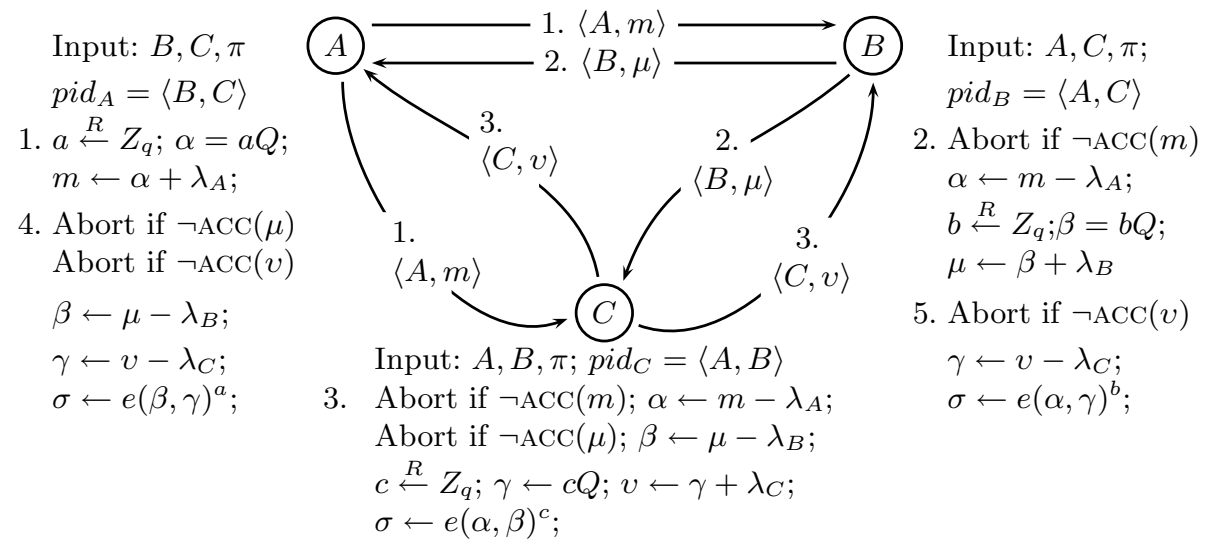

Fig. 1. Tripartite PPK protocol. Session ID is sid $=A\|B\| C\|m\| \mu \| v$. Shared session key is $s k=H\left(\left\langle A, B, C, m, \mu, v, \sigma, \lambda_{A}, \lambda_{B}, \lambda_{C}\right\rangle\right)$.

Table 1. Inputs and outputs for the participant queries

\begin{tabular}{|l|c|c|c|c|c|c|}
\hline & \multicolumn{2}{|c|}{$A$ (initiator) } & \multicolumn{3}{c|}{$B$ (second participant) } & \multicolumn{2}{c|}{ (third participant) } \\
\cline { 2 - 7 } & Input & Output & Input & Output & Input & Output \\
\hline Action 0 & $\langle B, C\rangle$ & $\langle A, m\rangle$ & $\langle A, m\rangle$ & $\langle B, \mu\rangle$ & $\langle A, m, B, \mu\rangle$ & $\langle C, v\rangle$ \\
\hline Action 1 & $\langle B, \mu, C, v\rangle$ & & $\langle C, v\rangle$ & & & \\
\hline
\end{tabular}


Let $\kappa$ be the cryptographic security parameter with $|q|=\kappa$. We use an elliptic curve $E$ over the integers modulo $p$ with coefficients $a, b$ in standard Weiestrass form and $\# E=r q$, with $\operatorname{gcd}(r, q)=1$. (Currently, $|p|=162$ and $|q|=160$ would be considered reasonably secure [9]). The complete specification is below:

$$
\text { sid } \leftarrow \text { pid } \leftarrow s k \leftarrow \varepsilon ; \text { acc } \leftarrow \text { term } \leftarrow F A L S E
$$

if state $=\operatorname{READY}$ and $(U \in I$ AND $U$ is the initiator) then

$\{$ Participant $A$ Action 0$\}\langle A\rangle \leftarrow U ;\langle B, C\rangle \leftarrow m s g-i n$ where $B, C \in I$;

$a \stackrel{R}{\leftarrow} Z_{q} ; \alpha=a Q ; \lambda_{A}=r \cdot f_{1}(A, B, C, \pi) ; m \leftarrow \alpha+\lambda_{A} ;$ state $\leftarrow\left\langle A, a, m, \lambda_{A}\right\rangle ;$ $m s g-$ out $\leftarrow\langle A, m\rangle$; return (msg-out, acc, term, sid,pid, sk, state)

elseif state $=$ READY and $(U \in I$ AND $U$ is the second participant $)$ then

$\{$ Participant $B$ Action 0$\}\langle B\rangle \leftarrow U ;\langle A, m\rangle \leftarrow m s g-i n$, where $A \in I$ and $\operatorname{ACCeptable}(m) ; \lambda_{A}=r \cdot f_{1}(A, B, C, \pi) ; \alpha \leftarrow m \leftarrow \lambda_{A} ; b \stackrel{R}{\leftarrow} Z_{q} ; \beta=b Q ;$ $\lambda_{B}=r \cdot f_{2}(A, B, C, \pi) ; \mu \leftarrow \beta+\lambda_{B} ;$ state $\leftarrow\left\langle A, a, m, B, b, \mu, \lambda_{A}, \lambda_{B}\right\rangle$, $m s g-$ out $\leftarrow\langle B, \mu\rangle$; return (msg-out, acc, term, sid, pid, sk, state)

elseif state $=$ READY and $(U \in I$ AND $U$ is the third participant) then

$\{$ Participant $C$ ACtion 0$\}\langle C\rangle \leftarrow U ;\langle A, m, B, \mu\rangle \leftarrow m s g-i n$, where $A, B \in I$ and $\operatorname{acceptable}(m)$ and $\operatorname{Acceptable}(\mu) ; \lambda_{A}=r \cdot f_{1}(A, B, C, \pi)$; $\alpha \leftarrow m-\lambda_{A} ; \lambda_{B}=r \cdot f_{2}(A, B, C, \pi) ; \beta \leftarrow \mu-\lambda_{B} ; c \stackrel{R}{\leftarrow} Z_{q} ; \gamma=c Q ;$ $\lambda_{C}=r \cdot f_{3}(A, B, C, \pi) ; v \leftarrow \gamma+\lambda_{C} ; \sigma \leftarrow e(\alpha, \beta)^{c} ;$ state $\leftarrow$ DONE; $m s g-$ out $\leftarrow\langle C, v\rangle ;$ sid $\leftarrow A\|B\| C\|m\| \mu \| v ;$ pid $\leftarrow\langle A, B\rangle ;$ sk $\leftarrow$ $H\left(\left\langle A, B, C, m, \mu, v, \sigma, \lambda_{A}, \lambda_{B}, \lambda_{C}\right\rangle\right) ;$ acc $\leftarrow$ term $\leftarrow$ TRUE; return $(m s g-$ out, acc, term, sid, pid, sk, state)

elseif state $=\left\langle A, a, m, \lambda_{A}\right\rangle$ and $(U \in I$ AND $U$ is the initiator) then

$\{$ Participant $A$ ACtion 1$\}\langle B, \mu, C, v\rangle \leftarrow m s g-i n$, where $B, C \in I$ and $\operatorname{ACCeptable}(\mu)$ and $\operatorname{ACCeptable}(v) ; \lambda_{B}=r \cdot f_{2}(A, B, C, \pi) ; \lambda_{C}=$ $r \cdot f_{3}(A, B, C, \pi) ; \beta \leftarrow \mu-\lambda_{B} ; \gamma \leftarrow v-\lambda_{C} ; \sigma \leftarrow e(\beta, \gamma)^{a} ;$ state $\leftarrow$ $D O N E ; m s g-$ out $\leftarrow \varepsilon ;$ sid $\leftarrow A\|B\| C\|m\| \mu \| v ;$ pid $\leftarrow(\langle B, C\rangle) ;$ sk $\leftarrow$ $H\left(\left\langle A, B, C, m, \mu, v, \sigma, \lambda_{A}, \lambda_{B}, \lambda_{C}\right\rangle\right) ;$ acc $\leftarrow$ term $\leftarrow$ TRUE; return $(m s g-$ out, acc, term, sid, pid, sk, state)

elseif state $=\left\langle A, a, m, B, b, \mu, \lambda_{A}, \lambda_{B}\right\rangle$ and $(U \in I$ AND $U$ is the second participant) then

$\{$ Participant $B$ ACtion 1$\}\langle C, v\rangle \leftarrow m s g-i n$, where $C \in I$ and AC$\operatorname{Ceptable}(v) ; \lambda_{C}=r \cdot f_{3}(A, B, C, \pi) ; \gamma \leftarrow v-\lambda_{c} ; \sigma \leftarrow e(\alpha, \gamma)^{b} ;$ state $\leftarrow$ $D O N E ;$ msg - out $\leftarrow \varepsilon ;$ sid $\leftarrow A\|B\| C\|m\| \mu \| v ;$ pid $\leftarrow\langle A, C\rangle ;$ sk $\leftarrow$ $H\left(\left\langle A, B, C, m, \mu, v, \sigma, \lambda_{A}, \lambda_{B}, \lambda_{C}\right\rangle\right) ;$ acc $\leftarrow$ term $\leftarrow$ TRUE; return $(m s g-$ out, acc, term, sid, pid, sk, state)

\section{Security of the Protocol}

Here we prove that the tripartite PPK protocol is secure, in the sense that an adversary attacking the system cannot determine session keys of fresh instances with greater advantage than that of an online dictionary attack.

Theorem 2. Let $P$ be the protocol described in Fig. 1] (and formally described above), using groups $G_{1}$ and $G_{2}$ of order $q$, with a password dictionary of size $N$. 
Fix an adversary $\mathcal{A}$ that runs in time $t$, and makes $n_{\text {se }}, n_{\text {ex }}$, and $n_{\text {re }}$ queries of type Send, Execute, and Reveal, respectively, and $n_{\text {ro }}$ queries to the random oracles. Let $t_{o p}$ be the time required to perform a scalar multiplication and a pairing of elliptic curve point in $G_{1}$ and an exponentiation in $G_{2}$. Then for $t^{\prime}=O\left(t+\left(n_{r_{o}}^{3}+n_{s e}+n_{e x}\right) t_{o p}\right):$

$$
A d v_{P}^{a k e}(\mathcal{A})=\frac{2 n_{s e}}{N}+O\left(A d v_{G_{1} G_{2}}^{B D H}\left(t^{\prime}, n_{r o}^{3}\right)+\frac{\left(n_{s e}+n_{e x}\right)\left(n_{r o}+n_{s e}+n_{e x}\right)}{q}\right)
$$

Proof. The proof proceeds by introducing a series of protocols $P_{0}, P_{1}, \cdots, P_{8}$ related to $P$, with $P_{0}=P$. In $P_{8}, \mathcal{A}$ is reduced to a simple online guessing attack that admits straightforward analysis. For each $i$ from 1 to 8 , we will prove that the advantage of $\mathcal{A}$ attacking protocol $P_{i-1}$ is at most negligibly more than the advantage of $\mathcal{A}$ attacking protocol $P_{i}$. An informal description of the protocols as well as a brief description in brackets of the basis for each proof follows:

$P_{0}$ The original protocol $P$.

$P_{1}$ If honest parties choose $m, \mu$, or $v$ values used previously in the protocol, it halts and $\mathcal{A}$ fails. (The probability of collision of nonces is negligible.)

$P_{2}$ The protocol answers Send and Execute queries without making any random oracle queries. Subsequent queries by $\mathcal{A}$ are backpatched, as much as possible, to be consistent with responses to Send and Execute queries. (This is consistent with $P_{1}$ unless $\mathcal{A}$ guesses the output of $f_{i}\left(A, B, C, \pi_{A B C}\right)$ queries correctly and uses the guesses in $H(\cdot)$ queries (for $i \in\{1,2,3\}$ ). However, the probability of correctly guessing the outputs is negligible.)

$P_{3}$ If an $H(\cdot)$ query is made, it is not checked for consistency against Execute queries. That is, instead of backpatching to maintain consistency with an Execute query, the protocol responds with a random output. (An instance of the BDH problem can be embedded in the $m \mu$ and $v$ values so that if backpatching would have been necessary, $H(\cdot)$ query inputs can be used to solve the BDH problem.)

$P_{4}$ If a correct password guess is made against any participant instance (determined by an $H(\cdot)$ query using the correct inputs to compute a session key), the protocol halts and $\mathcal{A}$ automatically succeeds. (This is obvious.)

$P_{5}$ If the adversary makes three password guesses against a Participant $A$ instance, the protocol halts and $\mathcal{A}$ fails. (This is shown by embedding an instance of the $\mathrm{BDH}$ problem in the $f_{i}$ queries and $A$ 's output. Inputs to the three $H(\cdot)$ queries used to find the session keys can be used to solve the $\mathrm{BDH}$ problem.)

$P_{6}$ and $P_{7}$ are similar to $P_{5}$, but for $B$ and $C$ respectively, instead of $A$.

$P_{8}$ The protocol uses an internal password oracle that holds all passwords and only accepts simple queries that test whether a given password is correct password for a given three parties. The test for correct password guesses (from $P_{4}$ ) is changed so that whenever the adversary makes a password guess, a query is submitted to the oracle to determine if it is correct. (By inspection $P_{7}$ and $P_{8}$ are indistinguishable.) 
We assume without loss of generality that $n_{r o}$ and $n_{s e}+n_{e x}$ are both at least 1. We make the standard assumption that random oracles are built "on the fly," that is, each new query to a random oracle is answered with a fresh random output, and each query that is not new is answered consistently with the previous queries. We also assume that the $f_{j}(\cdot)$ query is answered in the following way:

In an $f_{j}(A, B, C, \pi)$ query for $j \in\{1,2,3\}$, output $\phi_{j}[A, B, C, \pi] Q$, where $\quad \phi_{j}[A, B, C, \pi] \stackrel{R}{\longleftarrow} Z_{q}$. Also put $\quad \psi_{j}[A, B, C, \pi]=r \phi_{j}[A, B, C, \phi]$, $\lambda_{A} \leftarrow r \phi_{1}[A, B, C, \pi] Q, \quad \lambda_{B} \leftarrow r \phi_{2}[A, B, C, \pi] Q, \quad$ and $\quad \lambda_{C} \leftarrow r \phi_{3}[A, B, C, \pi] Q$. Denote $\psi_{j}[A, B, C, \pi]$ and $\phi_{j}[A, B, C, \pi]$ as $\psi_{j}[\pi]$ and $\phi_{j}[\pi]$ respectively. Thus $\psi_{j}[\pi]=r \phi_{j}[\pi]$.

We now define some events, corresponding to the adversary making a password guess against a participant instance, and against three participant instances that are partnered in an Execute query. In each case, we also define an associated value for the event, and we note that the associated value is actually fixed by the protocol before the event occurs.

testpw $(U, i, V, W, \pi)$ : This is the event that the adversary makes a password guess against $\prod_{i}^{U}$ with pid $_{U}=\langle V, W\rangle$. Let $\{U, V, W\}=\{A, B, C\}$ where $A$ is initiator, $B$ is the second participant and $\mathrm{C}$ is the third participant. For some $m, \mu, v, \lambda_{A}, \lambda_{B}$, and $\lambda_{C}, \mathcal{A}$ makes an $H\left(\left\langle A, B, C, m, \mu, v, \sigma, \lambda_{A}, \lambda_{B}, \lambda_{C}\right\rangle\right)$ query, and if $U=A, \mathcal{A}$ makes a Participant $U$ ACtion 0 query with input $\langle B, C\rangle$ and output $\langle A, m\rangle$, and a PARTicipant $U$ ACTION 1 query with input $\langle B, \mu, C, v\rangle$ to $\prod_{i}^{U}$. Otherwise, if $U=B, \mathcal{A}$ makes ParticIPANT $U$ ACTION 0 query with input $\langle A, m\rangle$ and output $\langle B, \mu\rangle$, and a PARTICIPANT $U$ ACTION 1 query with input $\langle C, v\rangle$ to $\prod_{i}^{U}$. Otherwise, since $U=C, \mathcal{A}$ makes Participant $U$ Action 0 query with input $\langle A, m, B, \mu\rangle$ and output $\langle C, v\rangle$ to $\prod_{i}^{U}$. $\mathcal{A}$ also makes an $f_{1}(A, B, C, \pi)$ query returning $\phi_{1}[\pi] Q$, an $f_{2}(A, B, C, \pi)$ query returning $\phi_{2}[\pi] Q$, an $f_{3}(A, B, C, \pi)$ query returning $\phi_{3}[\pi] Q$, where $\sigma=\operatorname{BDH}(\alpha, \beta, \gamma), m=\alpha+\lambda_{A}, \mu=\beta+\lambda_{B}$, $v=\gamma+\lambda_{C}, \lambda_{A}=\psi_{1}[\pi] Q, \lambda_{B}=\psi_{2}[\pi] Q, \lambda_{C}=\psi_{3}[\pi] Q$, ACCEPtable $(m)$, ACCEPTABLE $(\mu)$ and ACCEPTABLE $(v)$. The event's associated value is $s k_{U}^{i}=H\left(\left\langle A, B, C, m, \mu, v, \sigma, \lambda_{A}, \lambda_{B}, \lambda_{C}\right\rangle\right)$.

testexecpw $(A, i, B, j, C, l, \pi)$ : This is the event that the adversary makes a password guess against three instances that are partnered in an Execute query. For some $m, \mu, v, \lambda_{A}, \lambda_{B}$ and $\lambda_{C}, \mathcal{A}$ makes an $H\left(\left\langle A, B, C, m, \mu, v, \sigma, \lambda_{A}\right.\right.$, $\left.\left.\lambda_{B}, \lambda_{C}\right\rangle\right)$ query, and previously $\mathcal{A}$ made an Execute $(A, i, B, j, C, l)$ query that generated $m, \mu, v$, and $f_{1}(A, B, C, \pi), f_{2}(A, B, C, \pi)$, and $f_{3}(A, B, C, \pi)$ queries returning $\phi_{1}[\pi] Q, \phi_{2}[\pi] Q$, and $\phi_{2}[\pi] Q$, where $\lambda_{A}=\psi_{1}[\pi] Q, \lambda_{B}=$ $\psi_{2}[\pi] Q, \lambda_{C}=\psi_{3}[\pi] Q, \sigma=\mathbf{B D H}(\alpha, \beta, \gamma), m=\alpha+\lambda_{A}, \mu=\beta+\lambda_{B}$, and $v=\gamma+\lambda_{C}$. The associated value of this event is $s k_{A}^{i}=s k_{B}^{j}=s k_{C}^{l}=$ $H\left(\left\langle A, B, C, m, \mu, v, \sigma, \lambda_{A}, \lambda_{B}, \lambda_{C}\right\rangle\right)$.

correctpw: A testpw $\left(U, i, V, W, \pi_{U V W}\right)$ event occurred, for some $U, i, V, W$, where $\pi_{U V W}$ is the password shared between $U, V$, and $W$.

correctpwexec: A testexecpw $\left(A, i, B, j, C, l, \pi_{A B C}\right)$ event occured for $A, i$, $B, j, C$, and $l$, where $\pi_{A B C}$ is the password shared between $A, B$, and $C$. 
triplepw $(U):$ A testpw $(U, i, V, W, \pi)$ event, a testpw $(U, i, V, W, \hat{\pi})$ event and a $\operatorname{testpw}(U, i, V, W, \tilde{\pi})$ occurred, for some $U, i, V, W, \pi, \hat{\pi}$, and $\tilde{\pi}$ with $\pi \neq \hat{\pi} \neq$ $\tilde{\pi} \neq \pi$.

Protocol $P_{1}$. Let $E_{1}$ be the event that an $m$ value generated in a PARTICIPANT $A$ ACtion 0 or Execute query is equal an $m$ value generated in a previous PARTICIPANT $A$ ACtion 0 or Execute query, an $m$ value sent as input in a previous Participant $B$ Action 0, or Participant $C$ Action 0 query, or $m$ in a previous $f_{i}(\cdot)$ query (made by the adversary). Let $E_{2}$ be the event that a $\mu$ value generated in a PARTICIPANT $B$ ACTION 0 or Execute query is equal to a $\mu$ value generated in a previous PARTICIPANT $B$ ACtion 0 or Execute query, a $\mu$ sent as input in a previous Participant $A$ Action 1 or Participant $C$ ACTION 0 , or $\mu$ value in a previous $f_{j}(\cdot)$ query (made by the adversary). Let $E_{3}$ be the event that a $v$ value generated in a PARTicipant $C$ ACTion 0 or Execute query is equal to a $v$ value generated in a previous PARTICIPANT $C$ ACtion 0 or Execute query, a $v$ sent as input in a previous PARTICIPANT $A$ ACtion 1 or PARTiCipant $B$ ACtion 1 query, or $v$ value in a previous $f_{j}(\cdot)$ query (made by the adversary). Let $E=E_{1} \vee E_{2} \vee E_{3}$. Let $P_{1}$ be a protocol that is identical to $P_{0}$ except that if $E$ occurs, the protocol aborts (and thus the adversary fails).

Theorem 3. For an adversary $\mathcal{A}$,

$$
A d v_{P_{0}}^{a k e}(\mathcal{A}) \leq A d v_{P_{1}}^{a k e}(\mathcal{A})+\frac{O\left(\left(n_{s e}+n_{e x}\right)\left(n_{r o}+n_{s e}+n_{e x}\right)\right)}{q} .
$$

Proof. Consider the last $m, \mu$, or $v$ value generated. There is a probability of no more than $\frac{n_{r o}+n_{s e}+n_{e x}}{q}$ that this value has previously been generated in a Send, Execute or Random oracle query. There are $n_{s e}+n_{e x}$ values that are required to be unique if event $E$ is not to occur. Hence the probability of any of the $m, \mu$, or $v$ values not being unique is $\frac{O\left(\left(n_{s e}+n_{e x}\right)\left(n_{s e}+n_{e x}+n_{r o}\right)\right)}{q}$, and the theorem follows.

Protocol $P_{2}$. Let $P_{2}$ be a protocol that is identical to $P_{1}$ except that Send and Execute queries are answered without making any random oracle queries, and subsequent random oracle queries by the adversary are backpatched, as much as possible, to be consistent with the responses to the Send and Execute queries. Specifically, the queries in $P_{2}$ are changed as follows:

Execute $(A, i, B, j, C, l): m \leftarrow \tau[i, A] Q$, where $\tau[i, A] \stackrel{R}{\longleftarrow} Z_{q}, \mu \leftarrow \tau[j, B] Q$, where $\tau[j, B] \stackrel{R}{\longleftarrow} Z_{q}, v \leftarrow \tau[l, C] Q$, where $\tau[l, C] \stackrel{R}{\longleftarrow} Z_{q}$ and $s k_{A}^{i} \leftarrow s k_{B}^{j} \leftarrow$ $s k_{C}^{l} \stackrel{R}{\longleftarrow}\{0,1\}^{\kappa}$.

Participant $A$ ACtion 0 to $\prod_{i}^{A}: m \leftarrow \tau[i, A] Q$, where $\tau[i, A] \stackrel{R}{\leftarrow} Z_{q}$.

Participant $B$ ACtion 0 to $\Pi_{j}^{B}: \mu \leftarrow \tau[j, B] Q$, where $\tau[j, B] \stackrel{R}{\leftarrow} Z_{q}$.

Participant $C$ Action 0 to $\Pi_{l}^{C}: v \leftarrow \tau[l, C] Q$, where $\tau[l, C] \stackrel{R}{\leftarrow} Z_{q}$, and $s k_{C}^{l} \stackrel{R}{\longleftarrow}\{0,1\}^{\kappa}$. 
Participant $A$ ACtion 1 to $\prod_{i}^{A}$ : if $\prod_{l}^{C}$ is paired with instance $\prod_{i}^{A}$ and $\prod_{j}^{B}$, $s k_{A}^{i} \leftarrow s k_{B}^{j} \leftarrow s k_{C}^{l}$, else if $\prod_{l}^{C}$ is paired with instance $\prod_{i}^{A}, s k_{A}^{i} \leftarrow s k_{C}^{l}$, else if $\prod_{j}^{B}$ is paired with instance $\prod_{i}^{A}$ and have a session key $s k_{B}^{j}, s k_{A}^{i} \leftarrow s k_{B}^{j}$, else if this query causes a $\operatorname{testpw}\left(A, i, B, C, \pi_{A B C}\right)$ event to occur, set $s k_{A}^{i}$ to the value associated with that event, else set $s k_{A}^{i} \stackrel{R}{\leftarrow}\{0,1\}^{\kappa}$.

Participant $B$ ACtion 1 to $\prod_{j}^{B}$ : if $\prod_{l}^{C}$ is paired with instance $\prod_{i}^{A}$ and $\prod_{j}^{B}$, $s k_{A}^{i} \leftarrow s k_{B}^{j} \leftarrow s k_{C}^{l}$, else if $\prod_{l}^{C}$ is paired with instance $\prod_{j}^{B}, s k_{B}^{j} \leftarrow s k_{C}^{l}$, else if $\prod_{i}^{A}$ is paired with instance $\prod_{j}^{B}$ and has a session key $s k_{A}^{i}, s k_{B}^{j} \leftarrow s k_{A}^{i}$, else if this query causes a $\operatorname{testpw}\left(B, j, A, C, \pi_{A B C}\right)$ event to occur, set $s k_{B}^{j}$ to the value associated with that event, else set $s k_{B}^{j} \stackrel{R}{\leftarrow}\{0,1\}^{\kappa}$.

$H\left(\left\langle A, B, C, m, v, \sigma, \lambda_{A}, \lambda_{B}, \lambda_{C}\right\rangle\right)$ : if this $H(\cdot)$ causes a testpw $\left(A, i, B, C, \pi_{A B C}\right)$, testpw $\left(B, j, A, C, \pi_{A B C}\right)$, testpw $\left(C, l, A, B, \pi_{A B C}\right)$, or testexecpw $\left(A, i, B, j, C, l, \pi_{A B C}\right)$ event to occur, output the associated value of that event, else output a random value from $\{0,1\}^{\kappa}$.

Note that we can determine whether the appropriate event occurred using the $\phi_{1}[\pi], \phi_{2}[\pi], \phi_{3}[\pi]$, and $\tau$ values. Also note that by $P_{1}$ and the fact that a participant instance that is paired with any participant $C$ instance copies the session key of the participant $C$ instance (or, if there is no paired participant $C$ instance, then it copies the key of its partner, if such a partner exists), there will never be more than one associated value that needs to be considered in the $H(\cdot)$ query.

Theorem 4. For any adversary $\mathcal{A}, A d v_{P_{1}}^{a k e}(\mathcal{A})=A d v_{P_{2}}^{a k e}(\mathcal{A})+\frac{O\left(n_{r_{o}}\right)}{q}$.

Proof. In $P_{1}$, participant instance $\prod_{l}^{C}$ creates a session key $s k_{C}^{l}$ that is uniformly chosen from $\{0,1\}^{\kappa}$, independent of anything that previously occurred, since the $H(\cdot)$ query that determines $s k_{C}^{l}$ is new. Also in $P_{1}$, for any participant $\mathrm{A}$ and $\mathrm{B}$ instances $\prod_{i}^{A}$ and $\prod_{j}^{B}$ that have had an Action 1 query, either:

1. exactly one instance $\prod_{l}^{C}$ is paired with $\prod_{i}^{A}$ and $\prod_{j}^{B}$, in which case $s k_{C}^{l}=s k_{A}^{i}=s k_{B}^{j}$, or

2. only one instance $\prod_{l}^{C}$ is paired with $\prod_{i}^{A}$ or $\prod_{j}^{B}$, in which case $s k_{A}^{i}=s k_{C}^{l}$ or $s k_{B}^{j}=s k_{C}^{l}$, or

3. no instance $\prod_{l}^{C}$ is paired with $\prod_{i}^{A}$ and/or $\prod_{j}^{B}$, and $\prod_{i}^{A}$ and $\prod_{j}^{B}$ may or may not be paired with each other. In both of these cases, either a testpw $\left(A, i, B, C, \pi_{A B C}\right)$ or testpw $\left(B, j, A, C, \pi_{A B C}\right)$ event occurs, and $s k_{A}^{i}$ or $s k_{B}^{j}$ is the value associated with that event (i.e. the output of the previous $H(\cdot)$ query associated with that event) or $s k_{A}^{i}$ and $s k_{B}^{j}$ are uniformly chosen from $\{0,1\}^{\kappa}$, independent of anything that previously occurred, since the $H(\cdot)$ query that determines $s k_{A}^{i}$ and $s k_{B}^{j}$ is new.

Finally, for any $H\left(\left\langle A, B, C, \cdot, \cdot, \cdot, \cdot, \lambda_{A}, \lambda_{B}, \lambda_{C}\right\rangle\right)$ query, either (1) it causes a testpw $\left(A, i, B, C, \pi_{A B C}\right)$, testpw $\left(B, j, A, C, \pi_{A B C}\right)$, testpw $\left(C, l, A, B, \pi_{A B C}\right)$, 
or testexecpw $\left(A, i, B, j, C, l, \pi_{A B C}\right)$ event to occur, in which case the output is the associated value of that event, (2) $\lambda_{A}=r \cdot f_{1}\left(A, B, C, \pi_{A B C}\right), \lambda_{B}=r$. $f_{2}\left(A, B, C, \pi_{A B C}\right)$, and $\lambda_{C}=r \cdot f_{3}\left(A, B, C, \pi_{A B C}\right)$, but the adversary has not made $f_{1}\left(A, B, C, \pi_{A B C}\right), f_{2}\left(A, B, C, \pi_{A B C}\right)$, and $f_{3}\left(A, B, C, \pi_{A B C}\right)$ queries, or (3) the output of $H(\cdot)$ query is uniformly chosen from $\{0,1\}^{\kappa}$, independent of anything that previously occurred, since this is a new $H(\cdot)$ query.

If the second case for the $H(\cdot)$ query described above occurs, $P_{1}$ may be inconsistent with $P_{2}$, since the key associated with the relevant session may need to have been returned by $P_{2}$, instead of a random value. However, the probability of the adversary correctly guessing the value of $\lambda_{A}, \lambda_{B}$, and $\lambda_{C}$ in an $H(\cdot)$ query is less than $\frac{1}{q}$. Thus the total probability of an $H(\cdot)$ query causing the second case above is bounded by $\frac{n_{r o}}{q}$. If this case never occurs, then $P_{2}$ is consistent with $P_{1}$.

Protocol $P_{3}$. Let $P_{3}$ be identical to $P_{2}$ except that in an $H(\langle A, B, C, m, \mu, v, \sigma$, $\left.\left.\lambda_{A}, \lambda_{B}, \lambda_{C}\right\rangle\right)$ query, there is no testexecpw $\left(A, i, B, j, C, l, \pi_{A B C}\right)$ event check.

Theorem 5. For any $\mathcal{A}$ running in time $t$, there is a $t^{\prime}=O\left(t+\left(n_{\text {ro }}+n_{\text {ex }} t_{\text {op }}\right)\right.$ such that $A d v_{P_{2}}^{a k e}(\mathcal{A}) \leq A d v_{P_{3}}^{a k e}(\mathcal{A})+2 A d v_{G_{1} G_{2}}^{B D H}\left(t^{\prime}, n_{\text {ro }}\right)$.

Proof. Let $E$ be the event that a correctpwexec event occurs. if $E$ does not occur, then $P_{2}$ and $P_{3}$ are indistinguishable. Let $\varepsilon$ be the probability that $E$ occurs when $\mathcal{A}$ is running against protocol $P_{2}$. Then $\operatorname{Pr}\left(\operatorname{Succ}_{P_{2}}^{\text {ake }}(\mathcal{A})\right) \leq$ $\operatorname{Pr}\left(\operatorname{Succ}_{P_{3}}^{a k e}(\mathcal{A})\right)+\varepsilon$, and thus by Fact 1 $A d v_{P_{2}}^{a k e}(\mathcal{A}) \leq A d v_{P_{3}}^{a k e}(\mathcal{A})+2 \varepsilon$.

Now we construct algorithm $\mathcal{D}$ to solve $\mathbf{B D H}$ by running $\mathcal{A}$ on a simulation of the protocol. Given $(X, Y, Z), \mathcal{D}$ simulates $P_{3}$ for $\mathcal{A}$ with these changes.

1. In an Execute $(A, i, B, j, C, l)$ query, set $m \leftarrow X+\rho_{i, A} Q, \quad \mu \leftarrow Y+\rho_{j, B} Q$, $v \leftarrow Z+\rho_{l, C} Q$, where $\rho_{i, A}, \rho_{j, B}, \rho_{l, C} \stackrel{R}{\leftarrow} Z_{1}$.

2. When $\mathcal{A}$ finishes, for every $H\left(\left\langle A, B, C, m, \mu, v, \sigma, \lambda_{A}, \lambda_{B}, \lambda_{C}\right\rangle\right)$ query, where $m, \mu$, and $v$ were generated in an $\operatorname{Execute}(A, i, B, j, C, l)$ query and a $f_{1}(A, B, C, \pi)$ query returned $\phi_{1}[\pi] Q$ and an $f_{2}(A, B, C, \pi)$ query returned $\phi_{2}[\pi] Q$, an $f_{3}(A, B, C, \pi)$ query returned $\phi_{3}[\pi] Q$, and $\lambda_{A} \leftarrow r \phi_{1}[\pi] Q, \lambda_{B} \leftarrow$ $r \phi_{2}[\pi] Q, \lambda_{C} \leftarrow r \phi_{3}[\pi] Q$, add $\sigma e(X, Z)^{\psi_{3}[\pi]-\rho_{l, C}} e\left(v,\left(\rho_{j, B}-\psi_{2}[\pi]\right) X+\left(\rho_{i, A}-\psi_{1}[\pi]\right) Y\right)^{-1}$. $e\left(v,\left(\rho_{j, B}-\psi_{2}[\pi]\right) Q\right)^{\psi_{1}[\pi]-\rho_{i, A}} e\left(\left(\rho_{j, B}-\psi_{2}[\pi]\right) X+\left(\rho_{i, A}-\psi_{1}[\pi]\right) Y, \psi_{3}[\pi] Q\right)$. $e\left(\left(\rho_{j, B}-\psi_{2}[\pi]\right) Q,\left(\rho_{j, A}-\psi_{1}[\pi]\right) Q\right)^{\psi_{3}[\pi]}$

to the list of possible values for $\operatorname{BDH}(X, Y, Z)$, where $\psi_{i}[\pi]=r \phi_{i}[\pi]$, for $i=1,2,3$.

This simulation is perfectly indistinguishable from $P_{3}$ until $E$ occurs, and in this case, $\mathcal{D}$ adds the correct $\mathbf{B D H}(X, Y, Z)$ to the list. After $E$ occurs the simulation may be distinguishable from $P_{3}$, but $E$ still occurs with probability $\varepsilon$. We assume $\mathcal{A}$ still follows the appropriate time and query bounds (or that the simulator stops $\mathcal{A}$ from exceeding them). $\mathcal{D}$ creates a list of size $n_{\text {ro }}$, and its advantage is 
$\varepsilon$. Let $t^{\prime}$ be the running time of $\mathcal{D}$, and note that $t^{\prime}=O\left(t+\left(n_{\text {ro }}+n_{\text {ex }}\right) t_{\text {op }}\right)$. The theorem follows from the fact that $A d v_{G_{1} G_{2}}^{B D H}(\mathcal{D}) \leq A d v_{G_{1} G_{2}}^{B D H}\left(t^{\prime}, n_{\text {ro }}\right)$.

Protocol $P_{4}$. Let $P_{4}$ be a protocol that is identical to $P_{3}$ except that if correctpw occurs then the protocol halts and the adversary automatically succeeds. ( $P_{3}$ already checks for a correctpw event, in the PARTICIPAnT $A$ or $B$ Action 1 query to determine if the session key has already been determined, and in the $H(\cdot)$ query, to see if the output has already been determined.)

Theorem 6. For any adversary $\mathcal{A}, A d v_{P_{3}}^{a k e}(\mathcal{A}) \leq A d v_{P_{4}}^{a k e}(\mathcal{A})$.

Proof. Obvious.

Note that in $P_{4}$, until correctpw occurs, an $H(\langle A, B, C, \cdot, \cdot, \cdot, \cdot, \cdot, \cdot, \cdot\rangle)$ query will output a value uniformly chosen from $\{0,1\}^{\kappa}$, and the session key for an unpaired client instance will be uniformly chosen from $\{0,1\}^{\kappa}$.

Protocol $P_{5}$. Let $P_{5}$ be a protocol that is identical to $P_{4}$ except that if triplepw $(A)$ occurs, the protocol halts and the adversary fails. We assume that when a query is made, the test for triplepw $(A)$ occurs before the test for correctpw.

Theorem 7. For any adversary $\mathcal{A}$ running in time $t$, there is a $t^{\prime}=O(t+$ $\left.\left(n_{\text {ro }}^{3}+n_{\text {se }}+n_{\text {ex }}\right) t_{\text {op }}\right)$ such that $A d v_{P_{4}}^{\text {ake }}(\mathcal{A}) \leq A d v_{P_{5}}^{\text {ake }}(\mathcal{A})+9 A d v_{G_{1} G_{2}}^{B D H}\left(t^{\prime}, n_{\text {ro }}^{3}\right)$.

Proof. Let $\varepsilon$ be the probability that the triplepw $(A)$ event occurs when $\mathcal{A}$ is running against protocol $P_{4}$. Then $\operatorname{Pr}\left(\operatorname{Succ}_{P_{4}}^{a k e}(\mathcal{A})\right) \leq \operatorname{Pr}\left(\operatorname{Succ}_{P_{5}}^{\text {ake }}(\mathcal{A})\right)+\varepsilon$, and thus by Fact 1] $A d v_{P_{4}}^{a k e}(\mathcal{A}) \leq A d v_{P_{5}}^{a k e}(\mathcal{A})+2 \varepsilon$.

Now we construct algorithm $\mathcal{D}$ to solve $\mathrm{BDH}$ by running $\mathcal{A}$ on a simulation of the protocol. Given $(X, Y, Z), \mathcal{D}$ simulates $P_{4}$ for $\mathcal{A}$ with these changes:

1. In an $f_{2}(A, B, C, \pi)$ query and $f_{3}(A, B, C, \pi)$ query, set $f_{2}(A, B, C, \pi)=\psi_{2}[\pi] Y+\psi_{2}^{\prime}[\pi] Q$, where $\psi_{2}^{\prime}[\pi] \stackrel{R}{\longleftarrow} Z_{q}$, $f_{3}(A, B, C, \pi)=\psi_{3}[\pi] Z+\psi_{3}^{\prime}[\pi] Q$, where $\psi_{3}^{\prime}[\pi] \stackrel{R}{\longleftarrow} Z_{q}$, and $\left(\psi_{2}[\pi], \psi_{3}[\pi]\right) \in_{R}\{(0,1),(2,0),(0,2)\}$.

2. In a PARticipant $A$ ACtion 0 query to a participant instance $\prod_{i}^{A}$ with input $\langle B, C\rangle$, set $m \leftarrow X+\rho_{i, A} Q$.

3. Tests for correctpw (from $P_{4}$ ) are not made.

4. For every triple of queries $H\left(\left\langle A, B, C, m, \mu, v, \sigma, \lambda_{1}, \lambda_{2}, \lambda_{3}\right\rangle\right)$, $H\left(\left\langle A, B, C, m, \mu, v, \hat{\sigma}, \hat{\lambda}_{1}, \hat{\lambda}_{2}, \hat{\lambda}_{3}\right\rangle\right), \quad H\left(\left\langle A, B, C, m, \mu, v, \tilde{\sigma}, \tilde{\lambda}_{1}, \tilde{\lambda}_{2}, \tilde{\lambda}_{3}\right\rangle\right)$ where AC$\operatorname{Ceptable}(\sigma), \operatorname{ACCeptable}(\hat{\sigma})$ and $\operatorname{ACCeptable}(\tilde{\sigma})$ are true, and there was a PARTiCiPAnt $A$ ACtion 0 query to a participant instance $\prod_{i}^{A}$ with input $\langle B, C\rangle$ and output $\langle A, m\rangle$, a PARTicipant $A$ ACtion 1 query to $\prod_{i}^{A}$ with input $\langle B, \mu, C, v\rangle$, an $f_{k}(A, B, C, \pi)$ query that returned $\lambda_{k}$, an $f_{k}(A, B, C, \hat{\pi})$ query that returned $\hat{\lambda}_{k}$, and an $f_{k}(A, B, C, \tilde{\pi})$ query that returned $\tilde{\lambda}_{k}$, for $k \in\{1,2,3\}$, add 


$$
\begin{aligned}
& \left(\sigma^{2} \hat{\sigma}^{-1} \tilde{\sigma}^{-1} e(\mu, v)^{r\left(2 \phi_{1}[\pi]-\phi_{1}[\hat{\pi}]-\phi_{1}[\tilde{\pi}]\right)} e(X, \mu)^{r\left(2 \phi_{3}[\pi]-\phi_{3}[\hat{\pi}]-\phi_{3}[\tilde{\pi}]\right)}\right. \\
& e(X, v)^{r\left(2 \phi_{2}[\pi]-\phi_{2}[\hat{\pi}]-\phi_{2}[\tilde{\pi}]\right)} e(Y, v)^{-2 r^{2}\left(\phi_{1}[\pi]-\phi_{1}[\hat{\pi}]\right)} e(Z, \mu)^{-2 r^{2}\left(\phi_{1}[\pi]-\phi_{1}[\tilde{\pi}]\right)} \\
& e(X, Y)^{-2 r^{2}\left(\phi_{3}[\pi]-\phi_{3}[\hat{\pi}]\right)} e(X, Z)^{2 r^{2}\left(\phi_{2}[\pi]-\phi_{2}[\tilde{\pi}]\right)} e(Y, Z)^{2 r^{2}\left(r \phi_{1}[\pi]-\rho_{i, A}\right)} \\
& e(Q, \mu)^{r \rho_{i, A}\left(2 \phi_{3}[\pi]-\phi_{3}[\hat{\pi}]-\phi_{3}[\tilde{\pi}]\right)-r^{2}\left(2 \phi_{1}[\pi] \phi_{3}[\pi]-\phi_{1}[\hat{\pi}] \phi_{3}[\hat{\pi}]-\phi_{1}[\tilde{\pi}] \phi_{3}[\tilde{\pi}]\right)}
\end{aligned}
$$

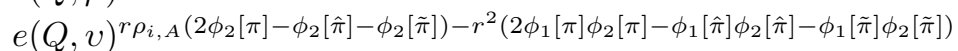

$$
\begin{aligned}
& e(Q, X)-r^{2}\left(2 \phi_{2}[\pi] \phi_{3}[\pi]-\phi_{2}[\hat{\pi}] \phi_{3}[\hat{\pi}]-\phi_{2}[\tilde{\pi}] \phi_{3}[\tilde{\pi}]\right) \\
& e(Q, Y)^{-2 r^{2} \rho_{i, A}\left(\phi_{3}[\pi]-\phi_{3}[\hat{\pi}]\right)+2 r^{3}\left(\phi_{1}[\pi] \phi_{3}[\pi]-\phi_{1}[\hat{\pi}] \phi_{3}[\hat{\pi}]\right)} \\
& e(Q, Z)^{-2 r^{2} \rho_{i, A}\left(\phi_{2}[\pi]-\phi_{2}[\tilde{\pi}]\right)+2 r^{3}\left(\phi_{1}[\pi] \phi_{2}[\pi]-\phi_{1}[\tilde{\pi}] \phi_{2}[\tilde{\pi}]\right)} \\
& e(Q, Q)^{-r^{2} \rho_{i, A}\left(2 \phi_{2}[\pi] \phi_{3}[\pi]-\phi_{2}[\hat{\pi}] \phi_{3}[\hat{\pi}]-\phi_{2}[\tilde{\pi}] \phi_{3}[\tilde{\pi}]\right)} \\
& e(Q, Q)^{\left.r^{3}\left(2 \phi_{1}[\pi] \phi_{2}[\pi] \phi_{3}[\pi]-\phi_{1}[\hat{\pi}] \phi_{2}[\hat{\pi}] \phi_{3}[\hat{\pi}]-\phi_{1}[\tilde{\pi}] \phi_{2}[\tilde{\pi}] \phi_{3}[\tilde{\pi}]\right)\right)^{\frac{1}{2 r^{2}}}}
\end{aligned}
$$

to the list of possible values of $\mathbf{B D H}(X, Y, Z)$.

This simulation is perfectly indistinguishable from $P_{4}$ until a triplepw $(A)$ event or a correctpw event occurs. If a triplepw $(A)$ event occurs, then with probability $\frac{2}{9}$ it occurs for three passwords $\pi, \hat{\pi}$, and $\tilde{\pi}$ with:

$$
\left\{\left(\psi_{2}[\pi], \psi_{3}[\pi]\right),\left(\hat{\psi}_{2}[\pi], \hat{\psi}_{3}[\pi]\right),\left(\tilde{\psi}_{2}[\pi], \tilde{\psi}_{3}[\pi]\right)\right\}=\{(1,1),(0,2),(2,0)\},
$$

in this case $\mathcal{D}$ adds the correct $\mathbf{B D H}(X, Y, Z)$ to the list. If a correctpw event occurs before a triplepw $(A)$ event occurs, then the triplepw $(A)$ event would never have occurred in $P_{4}$, since $P_{4}$ would halt. Note that in this case, the simulation may be distinguishable from $P_{4}$, but this does not change the fact that a triplepw $(A)$ event will occur with probability at least $\varepsilon$ in the simulation. However, we do make the assumption that $\mathcal{A}$ still follows the appropriate time and query bounds (or at least that the simulation can stop $\mathcal{A}$ from exceeding these bounds), even if $\mathcal{A}$ distinguished the simulation from $P_{4}$.

$\mathcal{D}$ creates a list of size less than $n_{r o}^{3}$, and its advantage is $\frac{2}{9} \varepsilon$. Let $t^{\prime}$ be the running time of $\mathcal{D}$, and note that $t^{\prime}=O\left(t+\left(n_{r_{o}}^{3}+n_{s e}+n_{e x}\right) t_{o p}\right)$. Then the theorem follows from the fact that $A d v_{G_{1} G_{2}}^{B H D}(\mathcal{D}) \leq A d v_{G_{1} G_{2}}^{B H D}\left(t^{\prime}, n_{\text {ro }}^{3}\right)$.

Protocol $P_{6}$. Let $P_{6}$ be a protocol that is identical to $P_{5}$ except that if triplepw $(B)$ occurs, the protocol halts and the adversary fails. We assume that when a query is made, the test for $\operatorname{triplepw}(B)$ occurs before the test for correctpw.

Theorem 8. For any adversary $\mathcal{A}$ running in time $t$, there is a $t^{\prime}=O(t+$ $\left.\left(n_{r_{o}}^{3}+n_{s e}+n_{e x}\right) t_{o p}\right)$ such that $A d v_{P_{5}}^{a k e}(A) \leq A d v_{P_{6}}^{a k e}(A)+9 A d v_{G_{1} G_{2}}^{B H D}\left(t^{\prime}, n_{r_{o}}^{3}\right)$

Proof. Omitted due to lack of space, but similar to that of Theorem 7

Protocol $P_{7}$. Let $P_{7}$ be a protocol that is identical to $P_{6}$ except that if triplepw $(C)$ occurs, the protocol halts and the adversary fails. We assume that when a query is made, the test for triplepw $(C)$ occurs before the test for correctpw.

Theorem 9. For any adversary $\mathcal{A}$ running in time $t$, there is a $t^{\prime}=O(t+$ $\left.\left(n_{r_{o}}^{3}+n_{s e}+n_{e x}\right) t_{o p}\right)$ such that $\operatorname{Adv}_{P_{6}}^{a k e}(\mathcal{A}) \leq A d v_{P_{7}}^{a k e}(\mathcal{A})+9 A d v_{G_{1} G_{2}}^{B H D}\left(t^{\prime}, n_{r_{o}}^{3}\right)$

Proof. Omitted due to lack of space, but similar to that of Theorem 7 
Protocol $P_{8}$. Let $P_{8}$ be a protocol that is identical to $P_{7}$ except that there is a new internal oracle (i.e., not available to the adversary) that handles passwords, called a password oracle. This oracle generates all passwords during initialization. Then it accepts queries of the form $\operatorname{testpw}(\pi)$ and returns TRUE if $\pi$ is correct, and FALSE otherwise. The protocol is changed only in the method for determining correctpw. Specifically, to test if correctpw occurs, whenever a testpw $(A, i, B, C, \pi)$, a testpw $(B, j, A, C, \pi)$ or a testpw $(C, l, A, B, \pi)$ event occurs, a testpw $(\pi)$ query is made to password oracle to see if $\pi$ is correct.

Theorem 10. For any adversary $\mathcal{A}, A d v_{P_{7}}^{a k e}(\mathcal{A})=A d v_{P_{8}}^{a k e}(\mathcal{A})$.

Proof. By inspection, $P_{7}$ and $P_{8}$ are perfectly indistinguishable.

The probability of the adversary $\mathcal{A}$ succeeding in $P_{8}$ is bounded by:

$\operatorname{Pr}\left(\operatorname{Succ}_{P_{8}}^{a k e}(\mathcal{A})\right) \leq \operatorname{Pr}($ correctpw $)+\operatorname{Pr}\left(\operatorname{Succ}_{P_{8}}^{\text {ake }}(\mathcal{A}) \mid \neg\right.$ correctpw $) \operatorname{Pr}(\neg$ correctpw $)$. First, since there are at most $2 n_{\text {se }}$ queries to the password oracle, and passwords are chosen uniformly from dictionary of size $\mathrm{N}, \operatorname{Pr}($ correctpw $) \leq \frac{2 n_{s e}}{N}$.

Now we compute $\operatorname{Pr}\left(\operatorname{Succ}_{P_{8}}^{\text {ake }}(\mathcal{A}) \mid \neg\right.$ correctpw $)$. If correctpw does not occur, then $\mathcal{A}$ succeeds by making a Test query to a fresh instance $\prod_{i}^{U}$ and guessing the bit used in that Test query. We will show that the view of the adversary is independent of $s k_{U}^{i}$, and thus the probability of success is exactly $\frac{1}{2}$.

First we examine Reveal queries. Recall that since $\prod_{i}^{U}$ is fresh, there could be no $\operatorname{Reveal}(U, i)$ query, and if $\prod_{i}^{U^{\prime}}$ is partnered with $\prod_{i}^{U}$, no $\boldsymbol{R e v e a l}\left(U^{\prime}, j\right)$ query. Second note that since sid includes $m$ and $\mu$ and $v$ values, if more than three participant instances accept with the same sid, $\mathcal{A}$ fails (see $P_{1}$ ). Thus the output of Reveal queries is independent of $s k_{U}^{i}$.

Second we examine $H(\cdot)$ queries. As noted in the discussion following the description of $P_{4}$, an $H(\cdot)$ query returns random values independent of anything that previously occurred. Thus any $H(\cdot)$ queries that occurs after $s k_{U}^{i}$ is set are independent of $s k_{U}^{i}$. But consider the following cases. (1) if $U$ is the third participant, $s k_{U}^{i}$ is chosen independently of anything that previously occurred (see $P_{2}$ ). (2) if $U$ is the first or second participant and is unpaired, $s k_{U}^{i}$ is chosen independently of anything that previously occurred (see the discussion after $P_{4}$ ). (3) if $U$ is the first or second participant and is paired, then $s k_{U}^{i} \leftarrow s k_{U^{\prime}}^{j} \leftarrow s k_{U^{\prime \prime}}^{l}$, where $\prod_{j}^{U^{\prime}}$ and $\prod_{l}^{U^{\prime \prime}}$ are the partners of $\prod_{i}^{U}$ and $U^{\prime \prime}$ is the third participant and $s k_{U^{\prime \prime}}^{l}$ is chosen independently of anything that previously occurred (see $P_{2}$ ). This implies that the adversary's view is independent of $s k_{U}^{i}$, and thus the probability of success is exactly $\frac{1}{2}$. Since $\operatorname{Pr}(\neg$ correctpw $)=1-\operatorname{Pr}($ correctpw $)$, we have:

$$
\begin{aligned}
\operatorname{Pr}\left(\text { Succ }_{P_{8}}^{\text {ake }}(\mathcal{A}) \leq \leq\right. & \operatorname{Pr}(\text { correctpw })+ \\
& \operatorname{Pr}\left(\operatorname{Succ}_{P_{8}}^{\text {ake }}(\mathcal{A}) \mid \neg \text { correctpw }\right) \operatorname{Pr}(\neg \text { correctpw }) \\
\leq & \operatorname{Pr}(\text { correctp } w))+\frac{1}{2}(1-\operatorname{Pr}(\text { correctp })) \\
\leq & \frac{1}{2}+\frac{\operatorname{Pr}(\text { correctp } w)}{2} \leq \frac{1}{2}+\frac{n_{\text {se }}}{N} .
\end{aligned}
$$

Therefore $A d v_{P_{7}}^{a k e} \leq \frac{2 n_{s e}}{N}$. Theorem 2 follows from this and Theorems 310 , 


\section{Complexity Comparison}

In this section we compare our protocol with Bresson et al.'s [7, which we call BCP. Because BCP is a group protocol, we fixed the group size to 3 for the comparison. Table 2 shows the total cost of computation and communication required. However, the three (symmetric) encryptions and three decryptions using the password as the key in BCP are not shown. Parings are the most expensive type of computation, and normally scalar multiplication on an elliptic curve is faster than exponentiation and finding a square root. We assume that each pairing computation is approximately equal to three exponentiations $[2,8$. Considering this assumption, the sum of pairings and exponentiations of our proposed protocol is about equal to the number of exponentiations of Bresson et al.'s protocol. Our protocol requires extra computations, i.e. 3 scalar multiplications and 18 square root computations. However, these can be pre-computed. In terms of message length, our protocol is better than BCP.

Table 2. The complexity of protocols

\begin{tabular}{|c|c|c|c|c|c|c|c|}
\hline \multirow{2}{*}{ Protocols } & \multicolumn{4}{|c|}{ Computation } & Communication & \\
\cline { 2 - 7 } & Pairings & $\begin{array}{l}\text { Scalar } \\
\text { Multipli- } \\
\text { cations }\end{array}$ & $\begin{array}{l}\text { Exponent- } \\
\text { iations }\end{array}$ & SQRT* & Passes & $\begin{array}{l}\text { Message } \\
\text { Length** }\end{array}$ & $\begin{array}{l}\text { Precomp- } \\
\text { utation }\end{array}$ \\
\hline Proposed & 3 & 3 & 3 & 18 & 3 & $3|p|$ & $\mathrm{Y}$ \\
\hline Bresson et. al.'s & - & - & 12 & & 3 & $9|g|$ & $\mathrm{N}$ \\
\hline
\end{tabular}

* Assume one application of $f_{i}(A, B, C, \pi)$ loops twice in MacKenzie's algorithm [15. ** $|p|$ is the bit size of finite field over which the elliptic curve is defined and $|g|$ is the bit size of the finite field over which the DLP is defined. When the base field is $\operatorname{GF}(2)$, normally $|p| \approx 250$ and $|g| \approx 1024$.

\section{Conclusion}

In this paper we proposed a provably secure one round password-based tripartite key agreement protocol, which builds on Joux's protocol and adapts the PAKEC scheme for password-based authentication. We proved the security of the proposed protocol using the random oracle model. It is better than an existing protocol by Bresson et al. in terms of message length. Although it requires extra computation compared to Bresson et al.'s, the extra part can be pre-computed.

Acknowledgements. We thank Colin Boyd and Juan Manuel González Nieto for many helpful suggestons and discussions. We also thank the anonymous referees of SAC 2005 for their comments.

\section{References}

1. S. Al-Riyami and K. Paterson, "Tripartite authenticated key agreement protocols from pairings," IMA Conference on Cryptography and Coding, LNCS vol. 2898, Springer-Verlag, pp.332-359. 2003. 
2. P. Barreto, H. Kim, and M. Scott, "Efficient algorithms for pairing-based cryptosystems," CRYPTO 2002, LNCS 2442, Springer-Verlag, pp.354-368, 2002.

3. M. Bellare, D. Pointcheval, and P. Rogaway, "Authenticated key exchange secure against dictionary attacks." In EUROCRYPT 2000, LNCS vol. 1807, pp.139-155, Springer-Verlag, 2000.

4. S. M. Bellovin and M. Merritt, "Encrypted key exchange: Password-based protocols secure against dictionary attacks," In IEEE Symposium on Research in Security and Privacy, pp.72-84, 1992.

5. S. Blake-Wilson, D. Johnson, and A. Menezese, "Key agreement protocols and their security analysis." In proceedings of the sixth IMA International Conferences on Cryptography and Coding, LNCS vol.1355, pp. 30-45, Springer-Verlag, 1997.

6. S. Blake-Wilson and A. Menezese, "Authenticated Diffie-Hellman key agreement protocols." In S. Tacares and H. Meijer, editors, Selected Areas in Cryptography (SAC'98), LNCS 1556, pp. 339-361, Springer-Verlag, 1998.

7. E. Bresson, O. Chevassut and D. Pointcheval, "Group Diffie-Hellman key exchange secure against dictionary attacks," Proceedings of Asiacrypt '02, LNCS vol. 2501, Springer-Verlag, pp.497-514, 2002.

8. S. Galbraith, K. Harrison and D. Soldera, "Implementing the Tate pairing," Algorithm Number Theory Symposium - ANTS V, LNCS vol. 2369, Springer-Verlag, pp. 324-337, 2002.

9. IEEE, IEEE1363 Standard Specifications for public key cryptography, 2000.

10. A. Joux, "A one round protocol for tripartite Deffie-Hellman." In W. Bosma, editor, Proceedings of Algorithmic Number Theory Symposium - ANTS IV, LNCS vol. 1838, pp.385-394, Springer-Verlag, 2000.

11. J. Kate, R. Ostrovsky, ans M. Young, "Practical password-authenticated key exchange provably secure under standard assumptions." In EUROCRYPT 2001, LNCS vol. 2045, pp.475-494, 2001

12. Y. Kim, A. Perrig and G. Tsudik, "Communication-efficient group key agreement," IFIP SEC 2001, Jun 2001.

13. L. Law, A. Menezes, M. Qu, J. Solinas, and S.A. Vanstone, "An efficient protocol for authenticated key agreement." Technical Report CORR 98-05, Department of C \& O, University of Waterloo, 1998.

14. L. Law, A. Menezes, M. Qu, J. Solinas, and S.A. Vanstone, "An efficient protocol for authenticated key agreement." Designs, Codes and Cryptography, vol. 28, no. 2, pp.119-134, 2003.

15. P. MacKenzie, "More efficient password-authenticated key exchange." CT-RSA 2001, LNCS vol. 2020, pp. 361-377, Springer-Verlag 2001.

16. P. MacKenzie, The PAK suit: Protocols for password-authenticated key exchange. DIMACS Technical report 2002-46, October 2002.

17. K. Shim, "Efficient one-round tripartite authenticated key agreement protocol from Weil pairing," Electronic Letters 39, pp.208-209, 2003.

18. F. Zhang, S. Liu and K. Kim, "ID-based one-round authenticated tripartite key agreement protocol with pairings," Cryptology ePrint archive, Report 2002/122. 